\title{
BMJ Open Optimal dose-fractionation schedule of palliative radiotherapy for patients with bone metastases: a protocol for systematic review and network meta- analysis
}

\author{
Xiaofang Tang, ${ }^{1}$ Qiancheng $\mathrm{Hu},{ }^{2}$ Ye Chen, ${ }^{2}$ Xin Wang, ${ }^{2}$ Xiaofen $\mathrm{Li},{ }^{2} \mathrm{Ke}$ Cheng, ${ }^{2}$ \\ Dan Cao (1) ${ }^{2}$
}

To cite: Tang $X$, Hu Q, Chen Y, et al. Optimal dose-fractionation schedule of palliative radiotherapy for patients with bone metastases: a protocol for systematic review and network meta-analysis. BMJ Open 2020;10:e033120. doi:10.1136/ bmjopen-2019-033120

- Prepublication history and additional material for this paper are available online. To view these files, please visit the journal online (http://dx.doi. org/10.1136/bmjopen-2019033120).

$\mathrm{XT}$ and $\mathrm{QH}$ are joint first authors.

Received 21 July 2019

Revised 01 December 2019

Accepted 16 December 2019

Check for updates

(C) Author(s) (or their employer(s)) 2020. Re-use permitted under CC BY-NC. No commercial re-use. See rights and permissions. Published by BMJ.

${ }^{1}$ Department of Emergency; Disaster Medical Center, Sichuan University West China Hospital, Chengdu, Sichuan, China

${ }^{2}$ Department of Abdominal Oncology, Cancer Center, Sichuan University West China Hospital, Chengdu, Sichuan, China

Correspondence to

Dr Dan Cao;

caodan316@163.com

\section{ABSTRACT}

Introduction The optimal dose-fractionation schedule of palliative radiotherapy has been debated in patients with bone metastases. Our objective is to comprehensively compare multiple fraction schedules with single fraction radiotherapy in terms of efficacy and toxicities by performing a systematic review and network metaanalysis.

Methods and analysis Electronic searches of titles/ abstracts of palliative radiotherapy for bone metastases will be performed, using PubMed, Cochrane Library, Embase, clinical trials, American Society for Therapeutic Radiology and Oncology and European Society of Radiotherapy and Oncology. The primary outcome of interest is the incidence of skeletal-related event following palliative radiotherapy for bone metastases in prospective studies. The risk of bias and quality of evidence will be evaluated based on Cochrane Collaboration's tool and Grades of Recommendation, Assessment, Development and Evaluation in the network meta-analysis. We will conduct subgroup analysis and sensitivity analysis regardless of heterogeneity estimates.

Ethics and dissemination This study will synthesise the evidence regarding dose-fractionation schedule of palliative radiotherapy in patients with bone metastases. We hope the findings from this study will help clinicians and patients select optimum palliative radiotherapy by identifying the optimal dose-fractionation schedule of palliative radiotherapy with the most value in terms of patient-important outcomes. The evidence obtained from network meta-analysis will help to guide head-to-head research in the future. The results will be disseminated through international conference reports and peerreviewed manuscripts. Ethics review board is not required for this network meta-analysis.

PROSPERO registration number CRD42019135195.

\section{INTRODUCTION}

Bone is the most frequent localisation where some tumours frequently develop secondary growth, with the incidence of over $85 \%$ patients with lung, breast or prostate
Strengths and limitations of this study

- Our objective is to comprehensively evaluate the evidence for palliative radiotherapy in patients with bone metastases and compare all multiple fraction schedules with single fraction radiotherapy in terms of efficacy and toxicities.

- We hope the findings from this study will help clinicians and patients select optimum palliative radiotherapy with the most value in terms of patientimportant outcomes in future.

- We will conduct a methodological and clinical heterogeneity study of a prespecified subgroup and also a sensitivity analysis to evaluate stability of the results in our network meta-analysis.

- The limitations of this network meta-analysis may be related to risk bias and quality of evidence, as the study includes not only randomised controlled trials (RCTs) but also non-RCTs.

cancers. $^{1-3}$ Generally, bone metastases are asymptomatic in some cases, but it has been confirmed that at least $75 \%$ of patients with cancer presenting symptomatic bone metastases. ${ }^{4}$ Bone metastases induced skeletalrelated events, for instance, pathological fractures, spinal cord compression and hypercalcaemia, impair quality of life and require multidisciplinary treatments. ${ }^{35}$

Palliative radiotherapy could have considerable impact on patients with asymptomatic and symptomatic bone metastases. ${ }^{67}$ Palliative radiotherapy induced ossification is the key to successfully relief cancer pain and kills tumour cells. ${ }^{8}$ In routine practice, five dose-fractionation schedules of radiotherapy, including 40 Gy (2 Gy/fraction),

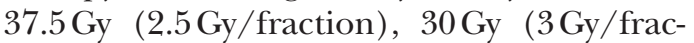
tion), $20 \mathrm{~Gy}$ (4Gy/fraction) and $8 \mathrm{~Gy}$ (single fraction), are indicated for the treatment 
of asymptomatic and symptomatic bone metastases; however, the optimal dose-fractionation scheme is a longlasting controversy with the paucity of evidence to make the best choice for each patient. ${ }^{9}$

Ratanatharathorn et al found that higher dose fractionated radiotherapy produced better outcomes in pain control, in terms of frequency, duration and magnitude, than low-dose regimens. ${ }^{10}$ However, the majority of metaanalyses produced the opposite results suggesting that the differences between single fraction and multiple fraction radiation treatment regimens were small and non-significant. ${ }^{11-16}$

There is little incentive for researchers to conduct active comparison trials for dose-fractionation schedule of palliative radiotherapy, due in part to the cost of clinical trials. Therefore, it is useful to synthesise evidence available from existing trials in dose-fractionation schedule of available palliative radiotherapy to compare the direct (based on previous trial comparisons) and indirect effects (palliative radiotherapy which are not previously identified in head-to-head comparisons). ${ }^{17-19}$ Our objective is to evaluate the evidence of the role of palliative radiotherapy for patients with bone metastases and, using a network meta-analysis, comprehensively compare all multiple fraction schedules with single fraction radiotherapy in terms of efficacy and toxicities.

\section{METHODS AND DESIGN}

The protocol of network meta-analysis will be prepared according to the recommendations of the Preferred Reporting Items for Systematic Reviews and Meta-Analyses Protocols (PRISMA).$^{20}$ We will report this system review and network meta-analysis according to the PRISMA Extension Statement for Reporting of Systematic Reviews Incorporating Network Meta-Analyses of Health Care Interventions. ${ }^{21}$

\section{Search strategy}

Electronic searches by titles/abstracts of palliative radiotherapy for bone metastases will be performed, using PubMed, Cochrane Library, Embase (Ovid interface) and clinical trials (www.clinicaltrials.gov/). Titles and abstracts referring to palliative radiotherapy for bone metastases will be searched in the electronic American Society for Therapeutic Radiology and Oncology and European Society of Radiotherapy and Oncology. A time frame from conception to 18 April 2019 will be applied for the database search. Two authors who are experienced in the information retrieval will conduct separate search strategies independently. We will manually search references of included related systematic reviews/meta-analyses and other relevant publications to identify additional potential studies. We will record the reason for excluding the full text and generate a PRIMSA flow diagram for the network meta-analysis. ${ }^{22}$

The search terms will include the following domains of Medical Subject Heading (MeSH) terms: 'bone and bones', 'neoplasm metastasis' and 'radiotherapy', according to Population Intervention Comparison Outcomes Study Design statement. MeSH and subheadings will be combined with 'AND' or 'OR'. A sample PubMed search strategy is described in details in (see online supplementary appendix).

We will perform a pilot test to evaluate inter-rater reliability and adjust each screening stage: title and abstract, followed by full-text screening. Independent reviewers will screen the titles/abstracts of related studies based on an inclusion and exclusion criteria. The eligible or potentially eligible trials will be assessed by reading through the full texts when necessary. Moreover, disagreements will be resolved by having a discussion, with the help of the third reviewer.

\section{Eligibility criteria}

The eligibility criteria must include the followings: (1) Symptomatic and asymptomatic bone metastases; (2) Interventions and Comparators: any dose-fractionation schedule of palliative radiotherapy, which is defined as alleviating focal symptoms of bone metastases or minimising the occurrence of skeletal related events with low total $\operatorname{dose}^{2324}$; (3) Randomised controlled trials (RCTs) and prospective studies; (4) Report or provide enough information to calculate ORs; (5) The outcomes are overall response of pain and the incidence of skeletal related event (pathological fracture, spinal cord compression, surgery to bone and hypercalcaemia) and grades 3-4 haematological and non-haematological toxicity; (6) There will be no restriction on language, status and year of publication and (7) The samples are not subject to any restriction, for example, age, gender, performance status, ethnicity or nationality. The excluding criteria are applied as follows: (1) reirradiation, brachytherapy, radiopharmaceuticals, particle irradiation, intraoperative irradiation, half-body irradiation or concurrent irradiation and chemotherapy; (2) Reviews, posters, abstracts, editorials and case reports and (3) cross-sectional, cohort, case-control or retrospective study designs.

\section{Outcomes}

The primary outcome of interest is overall response of pain and the incidence of skeletal related event (pathological fracture, spinal cord compression, surgery to bone and hypercalcaemia) ${ }^{25}$ following palliative radiotherapy for bone metastases in prospective studies. Overall response of pain is defined as the sum of partial response and complete response. Pain progression is defined as at least a two-point increase in pain scores without a reduction in analgesics, or a $\geq 25 \%$ increase in analgesics without a decrease in pain scores. The partial response is defined as at least a two-point reduction in pain scores without an increase in analgesics, or an analgesic intake decreased by more than $25 \%$ without an increase in pain scores. The complete response is defined as a pain score of zero (no pain) and no increasing morphine equivalent dose daily. Indeterminate response is defined as response 
that could not be classified according to pain progression, partial response or complete response definitions. ${ }^{12}$ The secondary outcomes are grades 3-4 haematological and non-haematological toxicity. The haematological toxicity is defined as anaemia, thrombocytopaenia, leucopenia and neutropaenia. For the response rates which are not reported in some trials, the percentages of responses will be recalculated according to the number of patients in the original studies.

\section{Data extraction and management}

The management of literature search records will be carried out in EndNote X7. A spreadsheet will be created in Microsoft Excel 2010 (Microsoft, Redmond, Washington, USA, www.microsoft.com) to collect outcomes of interest, such as the first author, study design, recruitment time frame, characteristics of bone metastases, details of interventions, sample size and endpoints (pain response, odds ratios and grades 3-4 haematological and non-haematological toxicity).

\section{Bias risk}

The quality and the risk of bias of RCTs will be estimated according to the following domains outlined in the Cochrane Collaboration's tool, ${ }^{27}$ which includes seven specific domains: random sequence generation, allocation concealment, blinding of participants and personnel, incomplete outcome data, selective outcome reporting, and other bias. RCTs will be independently reviewed by two authors and reported as high risk of bias '-', low risk of bias '+', or unclear risk of bias '?'. All nonRCTs will be assessed by Risk Of Bias In Non-Randomised Studies of Interventions guidelines. ${ }^{28}$ Any disagreement in assessment will be resolved via having a discussion, with the help of the third reviewer.

\section{Quality of evidence}

The quality of evidence in the network meta-analysis will be evaluated based on the Grades of Recommendation, Assessment, Development and Evaluation (GRADE), which include the following five domains: risk of bias, imprecision, inconsistency, indirectness and publication bias. ${ }^{29}$ The staging system categorises GRADE evidence into four stages: (1) high, (2) moderate, (3) low and (4) very low quality. For RCTs, the starting confidence level for each network estimate is high, but will be rated down based on the evaluation of the five domains. For observational studies, the starting confidence level for each network estimate is low, but will be rated up based on the evaluation of the three domains: large effect, plausible confounding and dose-response gradient. ${ }^{30}$ We will complete the GRADE process with GRADEprofiler software (GRADEpro, V.3.6.1) (available at: www.gradeworkinggroup.org).

\section{Direct comparison and network meta-analyses}

We will perform the traditional pairwise meta-analysis between each direct comparison, and generate graphics (network map, contribution plot, comparisons adjusted funnel plot, pairwise meta-analysis, estimation of inconsistency, local heterogeneity and surface under the cumulative ranking curve (SUCRA) graphs)) for network meta-analysis using Stata V.13.0 (StataCorp). A network plot will be constructed consisting of nodes and edges per outcome, and the weight of each node will be proportional to the number of patients. A contribution plot will be proposed to evaluate the contribution of direct and mixed interventions to the estimation of network metaanalytical summary effects. A comparison-adjusted funnel plot will be conducted to assess the potential publication bias of all included studies (if more than 10 studies are present). To compare all dose-fractionation schedule of palliative radiotherapy, network meta-analysis for all outcomes is planned using GeMTC V.1.4.3, (MRC Biostatistics Unit, Cambridge, UK). Results regarding the pain, skeletal related event, grades 3-4 haematological and non-haematological toxicity are expressed as ORs for dichotomous outcomes with $95 \%$ CIs/credible intervals. Both fixed and random-effects models will be run for pain, skeletal-related event, grades 3-4 haematological and non-haematological toxicity. ${ }^{31} \mathrm{We}$, thus, use the deviance information criterion (DIC), of which a difference of more than five points is considered significant, for Bayesian model selection. We will select the model with the lowest value of DIC to explain our results. ${ }^{32} 33$ A rough comparison will be performed between the fit of the inconsistency model with that of the consistency model. ${ }^{34} 35$ Node-splitting analysis and loop-specific approach will be used to assess inconsistency in each comparison into direct and indirect evidence and closed loop in network meta-analysis, respectively. ${ }^{36}$ When there is no obvious inconsistency, the consistency model will be used; otherwise, an inconsistent model will be used. ${ }^{36}$ The group with SUCRA of being the most effective in term of efficacy and safety will be evaluated based on the network meta-analysis results. According to SUCRA, we will rank the best probability of each intervention, followed by the second, the third and so on. We will perform a cluster analysis with SUCRA in terms of efficacy and tolerability. The extent of heterogeneity is assessed with the $\mathrm{I}^{2}$ statistic. If $\mathrm{I}^{2}>50 \%$, we estimate statistical heterogeneity as evidence of high; as moderate if $25 \% \leq \mathrm{I}^{2} \leq 50 \%$; as low if $\mathrm{I}^{2}<25 \% 0 .{ }^{38}$ Twosided $\mathrm{p}<0.05$ is considered significant.

\section{Transitivity, homogeneity and consistency assumption}

To achieve valid results, we will perform three key assumptions underlying the network meta-analysis (transitivity, homogeneity and consistency assumption). ${ }^{39}$ First, we will conduct a thorough comparison of important study and patient characteristics. Second, we will perform a multivariate metaregression analysis to examine possible sources of heterogeneity using the same interventions. Lastly, indirect evidence via a common comparator is not different from direct evidence in the network. ${ }^{26} 37$ 


\section{Subgroup and sensitivity analyses}

We will conduct prespecified subgroup analyses for our primary outcomes based on symptomatic and asymptomatic bone metastases. Symptomatic bone metastases are loosely defined as the use of palliative radiotherapy or surgical intervention to relieve pain, incident of new symptomatic pathological bone fractures and spinal cord compression, ${ }^{40}$ whereas asymptomatic bone metastases are defined as no bone pain and no evidence of pathological fracture or spinal cord compression. ${ }^{41}$ Additional subgroup analyses will be conducted, if possible, based on the cancer type (breast and prostate cancer vs others), site of bone metastases (vertebral metastasis vs limb metastasis) and publication year (before 2002 vs after 2002). ${ }^{42}$

A series of three sensitivity analyses will be conducted according to the impact of Bayesian model (fixed-effect model vs random-effect model), study design (RCTs vs prospective studies) and overall low risk of bias. Then, additional sensitivity analyses will be conducted by excluding one paper at a time and observing the robustness of the results. ${ }^{43}$

\section{DISCUSSION}

The high prevalence of bone metastases imposes a substantial socioeconomic burden, which attracts the attention of governments, pharmaceutical companies, academic researchers and other healthcare payers. More recent RCTs and non-RCTs have demonstrated the potential benefits of certain dose fractionations of palliative radiotherapy. ${ }^{6} 11$ 44-47 However, the results of publications focusing on palliative radiotherapy in patients with bone metastases varied significantly for study designs and varying outcomes of interest, especially for different dosefractionation schedule. The optimal dose-fractionation schedule of palliative radiotherapy in patients with bone metastases has long been debated, with multiple fraction schedules and single fraction radiotherapy in terms of efficacy, safety and cost-effectiveness. Accordingly, there are substantial practice changes among radiation oncologists treating bone metastases. Single fraction radiation remains frequently used in Europe, Canada and Australia, ${ }^{48-50}$ while multiple fraction radiation is likely advocated in the $\mathrm{USA}^{51}$ and China. ${ }^{52}$

To the best of our knowledge, the results of network meta-analysis of prospective trials will fill a crucial knowledge gap of optimal dose-fractionation schedule of palliative radiotherapy in patients with bone metastases. We hope the findings from this study will help clinicians and patients select optimum palliative radiotherapy in the future by identifying the optimal dose-fractionation schedule of palliative radiotherapy with the most value in terms of patient-important outcomes. Additionally, currently under-recognised palliative radiotherapy comparisons may be identified by system reviews and network meta-analysis to guide future research and headto-head RCTs.
Acknowledgements Thanks to Sun Feng and Wu Shanshan (PhD, Perking University School of public Health) for providing assistance and contributing to statistical analysis. (https://class.dxy.cn/)

Contributors $\mathrm{QH}$ and $\mathrm{DC}$ conceptualised the network meta-analysis. QH and XW codeveloped the search strategy. Both XT and QH were major contributors in writing the manuscript. The protocol was revised by DC,YC, XL and KC. DC was serving as guarantor and corresponding author of this study. All authors approved the final manuscript and agreed to submit the protocol in the journal.

Funding The network meta-analysis was supported by the National Natural Science Foundation of China (grant no. 81773097).

Competing interests None declared.

Patient consent for publication Not required.

Provenance and peer review Not commissioned; externally peer reviewed.

Open access This is an open access article distributed in accordance with the Creative Commons Attribution Non Commercial (CC BY-NC 4.0) license, which permits others to distribute, remix, adapt, build upon this work non-commercially, and license their derivative works on different terms, provided the original work is properly cited, appropriate credit is given, any changes made indicated, and the use is non-commercial. See: http://creativecommons.org/licenses/by-nc/4.0/.

ORCID iD

Dan Cao http://orcid.org/0000-0002-6709-4932

\section{REFERENCES}

1 Maroni P. Megakaryocytes in bone metastasis: protection or progression? Cells 2019;8:134.

2 Triedman SA, Radie-Keane K. The role of radiation therapy in the management of bone metastases. Med Health R / 1996;79:135-8.

3 De Felice F, Piccioli A, Musio D, et al. The role of radiation therapy in bone metastases management. Oncotarget 2017;8:25691-9.

4 Wagner G. Frequency of pain in patients with cancer. Recent Results Cancer Res 1984;89:64-71.

5 Aielli F, Ponzetti M, Rucci N. Bone metastasis pain, from the bench to the bedside. Int J Mol Sci 2019;20:280.

6 Wu JS-Y, Wong R, Johnston M, et al. Meta-Analysis of dosefractionation radiotherapy trials for the palliation of painful bone metastases. Int J Radiat Oncol Biol Phys 2003;55:594-605.

7 Wu JS-Y, Wong RKS, Lloyd NS, et al. Radiotherapy fractionation for the palliation of uncomplicated painful bone metastases - an evidence-based practice guideline. BMC Cancer 2004;4:71.

8 Goblirsch MJ, Zwolak PP, Clohisy DR. Biology of bone cancer pain. Clin Cancer Res 2006;12:6231s-5.

9 Rades D, Stalpers LJA, Veninga T, et al. Evaluation of five radiation schedules and prognostic factors for metastatic spinal cord compression. J Clin Oncol 2005;23:3366-75.

10 Ratanatharathorn V, Powers WE, Moss WT, et al. Bone metastasis: review and critical analysis of random allocation trials of local field treatment. Int J Radiat Oncol Biol Phys 1999;44:1-18.

11 Chow E, Harris K, Fan G, et al. Palliative radiotherapy trials for bone metastases: a systematic review. J Clin Oncol 2007;25:1423-36.

12 Chow E, Zeng L, Salvo N, et al. Update on the systematic review of palliative radiotherapy trials for bone metastases. Clin Oncol 2012;24:112-24.

13 Chow R, Hoskin P, Chan S, et al. Efficacy of multiple fraction conventional radiation therapy for painful uncomplicated bone metastases: a systematic review. Radiother Oncol 2017;122:323-31.

14 Rich SE, Chow R, Raman S, et al. Update of the systematic review of palliative radiation therapy fractionation for bone metastases. Radiother Oncol 2018;126:547-57.

15 McQuay HJ, Collins SL, Carroll D, et al. Radiotherapy for the palliation of painful bone metastases. Cochrane Database Syst Rev 2000:Cd001793.

16 Mizumoto M, Harada $\mathrm{H}$, Asakura $\mathrm{H}$, et al. Prognostic factors and a scoring system for survival after radiotherapy for metastases to the spinal column: a review of 544 patients at Shizuoka cancer center Hospital. Cancer 2008;113:2816-22.

17 Caldwell DM, Ades AE, Higgins JPT. Simultaneous comparison of multiple treatments: combining direct and indirect evidence. BMJ 2005;331:897-900.

18 Salanti G, Marinho V, Higgins JPT. A case study of multipletreatments meta-analysis demonstrates that covariates should be considered. J Clin Epidemiol 2009;62:857-64. 
19 Cipriani A, Higgins JPT, Geddes JR, et al. Conceptual and technical challenges in network meta-analysis. Ann Intern Med 2013;159:130-7.

20 Moher D, Shamseer L, Clarke M, et al. Preferred reporting items for systematic review and meta-analysis protocols (PRISMA-P) 2015 statement. Syst Rev 2015;4:1.

21 Hutton B, Salanti G, Caldwell DM, et al. The PRISMA extension statement for reporting of systematic reviews incorporating network meta-analyses of health care interventions: checklist and explanations. Ann Intern Med 2015;162:777-84.

22 Moher D, Liberati A, Tetzlaff J, et al. Preferred reporting items for systematic reviews and meta-analyses: the PRISMA statement. BMJ 2009;339:b2535.

23 Spencer K, Parrish R, Barton R, et al. Palliative radiotherapy. BMJ 2018;360:k821.

24 Nielsen OS. Palliative radiotherapy of bone metastases: there is now evidence for the use of single fractions. Radiother Oncol 1999;52:95-6.

25 So A, Chin J, Fleshner N, et al. Management of skeletal-related events in patients with advanced prostate cancer and bone metastases: incorporating new agents into clinical practice. Can Urol Assoc J 2012;6:465-70.

26 Tesfamariam Y, Jakob T, Wöckel A, et al. Adjuvant bisphosphonates or RANK-ligand inhibitors for patients with breast cancer and bone metastases: a systematic review and network meta-analysis. Crit Rev Oncol Hematol 2019;137:1-8.

27 Higgins JPT, Altman DG, Gøtzsche PC, et al. The Cochrane collaboration's tool for assessing risk of bias in randomised trials. BMJ 2011;343:d5928.

28 Sterne JA, Hernán MA, Reeves BC, et al. ROBINS-I: a tool for assessing risk of bias in non-randomised studies of interventions. BMJ 2016;355:i4919.

29 Atkins D, Eccles M, Flottorp S, et al. Systems for grading the quality of evidence and the strength of recommendations I: critical appraisal of existing approaches the grade Working group. BMC Health Serv Res 2004;4:38.

30 Hawkes N. Advantages of proton beam therapy in Ashya King's type of cancer are small, doctors say. BMJ 2014;349:g5610.

31 Cameron C, Coyle D, Richter T, et al. Systematic review and network meta-analysis comparing antithrombotic agents for the prevention of stroke and major bleeding in patients with atrial fibrillation. BMJ Open 2014;4:e004301.

32 Qiu Z, Song PXK, Tan M. Bayesian hierarchical models for multilevel repeated ordinal data using WinBUGS. J Biopharm Stat 2002;12:121-35.

33 Zintzaras E, loannidis JPA. Heterogeneity testing in meta-analysis of genome searches. Genet Epidemiol 2005;28:123-37.

34 Wieneke H, Naber CN, Piaszek L, et al. Better identification of patients who benefit from implantable cardioverter defibrillators by genotyping the G protein beta3 subunit (GNB3) C825T polymorphism. Basic Res Cardiol 2006;101:447-51.

35 Xu C, Chen Y-P, Du X-J, et al. Comparative safety of immune checkpoint inhibitors in cancer: systematic review and network metaanalysis. BMJ 2018;363:k4226.

36 Dias S, Welton NJ, Caldwell DM, et al. Checking consistency in mixed treatment comparison meta-analysis. Stat Med 2010;29:932-44.
37 Higgins JPT, Jackson D, Barrett JK, et al. Consistency and inconsistency in network meta-analysis: concepts and models for multi-arm studies. Res Synth Methods 2012;3:98-110.

38 Mao L, Jian C, Changzhi L, et al. Cytochrome CYP2C19 polymorphism and risk of adverse clinical events in clopidogreltreated patients: a meta-analysis based on 23,035 subjects. Arch Cardiovasc Dis 2013;106:517-27.

39 Song F, Loke YK, Walsh T, et al. Methodological problems in the use of indirect comparisons for evaluating healthcare interventions: survey of published systematic reviews. BMJ 2009;338:b1147.

40 Sartor O, Coleman R, Nilsson S, et al. Effect of radium-223 dichloride on symptomatic skeletal events in patients with castration-resistant prostate cancer and bone metastases: results from a phase 3 , double-blind, randomised trial. Lancet Oncol 2014;15:738-46.

41 Shulman RM, Meyer JE, Li T, et al. External beam radiation therapy (EBRT) for asymptomatic bone metastases in patients with solid tumors reduces the risk of skeletal-related events (SREs). Ann Palliat Med 2019;8:159-67.

42 Chow E, Wu JSY, Hoskin P, et al. International consensus on palliative radiotherapy endpoints for future clinical trials in bone metastases. Radiother Oncol 2002;64:275-80.

43 Zacher J, Kasenda B, Engert A, et al. The role of additional radiotherapy for primary central nervous system lymphoma. Cochrane Database Syst Rev 2014:CD009211.

44 Roos DE, Turner SL, O'Brien PC, et al. Randomized trial of 8 Gy in 1 versus 20 Gy in 5 fractions of radiotherapy for neuropathic pain due to bone metastases (Trans-Tasman radiation Oncology group, TROG 96.05). Radiother Oncol 2005;75:54-63.

45 Truntzer P, Atlani D, Pop M, et al. Early evaluation predicts pain relief of irradiated bone metastases: a single-center prospective study. BMC Palliat Care 2013;12:12.

46 Zeng L, Chow E, Bedard G, et al. Quality of life after palliative radiation therapy for patients with painful bone metastases: results of an international study validating the EORTC QLQ-BM22. Int J Radiat Oncol Biol Phys 2012;84:e337-42.

47 Caissie A, Zeng L, Nguyen J, et al. Assessment of health-related quality of life with the European organization for research and treatment of cancer QLQ-C15-PAL after palliative radiotherapy of bone metastases. Clin Oncol 2012;24:125-33.

48 McDonald R, Chow E, Lam H, et al. International patterns of practice in radiotherapy for bone metastases: a review of the literature. $J$ Bone Oncol 2014;3:96-102.

49 Olson RA, Tiwana MS, Barnes M, et al. Use of single- versus multiple-fraction palliative radiation therapy for bone metastases: population-based analysis of 16,898 courses in a Canadian Province. Int J Radiat Oncol Biol Phys 2014;89:1092-9.

50 Petrushevski AN, Gabriel GS, Hanna TP, et al. Factors affecting the use of single-fraction radiotherapy for the palliation of bone metastases in Australia. Clin Oncol 2015;27:205-12.

51 Rutter CE, Yu JB, Wilson LD, et al. Assessment of national practice for palliative radiation therapy for bone metastases suggests marked underutilization of single-fraction regimens in the United States. Int $J$ Radiat Oncol Biol Phys 2015;91:548-55.

$52 \mathrm{He} \mathrm{J}$, Zeng Z-C, Tang Z-Y, et al. Clinical features and prognostic factors in patients with bone metastases from hepatocellular carcinoma receiving external beam radiotherapy. Cancer 2009;115:2710-20. 